\title{
INDEFINITELY DIFFERENTIABLE FUNCTIONS WITH PRESCRIBED LEAST UPPER BOUNDS ${ }^{1}$
}

IRWIN E. PERLIN

1. Introduction. Let $F(x)$ be a real indefinitely differentiable function of the real variable $x$ defined on the interval $a \leqq x \leqq b$, and let $M_{n}$ denote the least upper bound of $\left|F^{(n)}(x)\right|$ on that interval. ${ }^{2}$ In this paper we shall establish sufficient conditions that there exist an indefinitely differentiable function $F(x)$ taking on certain prescribed $M_{n}$.

It is easy to see that $M_{0}$ and $M_{1}$ can be assigned arbitrarily. However, the first three upper bounds $M_{0}, M_{1}$, and $M_{2}$ must satisfy certain inequalities. ${ }^{3}$ Let us consider the interval $(0,1)$. Let $t_{1}$ be the value of $x$ for which $\left|F^{(1)}(x)\right|$ attains its maximum. Then

$$
F(1)-F\left(t_{1}\right)=\left(1-t_{1}\right) F^{(1)}\left(t_{1}\right)+(1 / 2 !) F^{(2)}\left(\theta_{1}\right)\left(1-t_{1}\right)^{2},
$$

where $t_{1}<\theta_{1}<1$. And similarly

$$
F(0)-F\left(t_{1}\right)=-t_{1} F^{(1)}\left(t_{1}\right)+(1 / 2 !) F^{(2)}\left(\theta_{2}\right) t_{1}^{2},
$$

where $0<\theta_{2}<t_{1}$. On subtracting these equations we obtain

$$
\begin{aligned}
F^{(1)}\left(t_{1}\right) & =F(1)-F(0)+(1 / 2 !)\left\{F^{(2)}\left(\theta_{2}\right) t_{1}^{2}-F^{(2)}\left(\theta_{1}\right)\left(1-t_{1}\right)^{2}\right\}, \\
M_{1} & \leqq 2 M_{0}+M_{2} / 2 ! .
\end{aligned}
$$

By the same procedure we can obtain for the interval $(0, a)$

$$
M_{1} \leqq 2 M_{0} / a+M_{2} a / 2 \text { !. }
$$

In the case of the interval $(0, \infty)$ we can replace (1) by a more precise inequality. Since $a$ is arbitrary, we can replace $a$ by the positive value which minimizes the right side of (1), and obtain

$$
M_{1} \leqq 2\left(M_{0} M_{2}\right)^{1 / 2} \text {. }
$$

Ore $^{4}$ in a recent paper employed the results of W. Markoff to obtain certain inequalities connecting the least upper bounds of $\left|F^{(i)}(x)\right|,(1 \leqq i \leqq n)$, with those of $|F(x)|$ and $\left|F^{(n+1)}(x)\right|$ where $F(x)$ is a function with bounded $(n+1)$ th derivative. For the first derivative the inequality (1) is slightly better than that obtained by Ore.

\footnotetext{
1 Presented to the Society, September 8, 1939.

${ }^{2}$ By $F^{(0)}(x)$ we shall mean $F(x)$.

${ }^{3}$ Hadamard, Comptes Rendus des Séances de la Société Mathématique de France, 1914, pp. 68-72; T. Carlman, Les fonctions quasi analytiques, Paris, 1926.

${ }^{4} \mathrm{O}$. Ore, On functions with bounded derivatives, Transactions of this Society, vol. 43 (1938), pp. 321-326.
} 
2. Construction of an indefinitely differentiable function with prescribed least upper bounds. We now prove the following theorem.

THEOREM. If the sequence $\left\{M_{i} a^{i}\right\}$ is monotone decreasing, then there exists an indefinitely differentiable function $F(x)$ defined on $(0 \leqq x \leqq a)$ such that $M_{i}$ is the least upper bound of $\left|F^{(i)}(x)\right|$ on $(0 \leqq x \leqq a)$.

Define

$$
0 \leqq S_{i}=\sum_{j=0}^{\infty}(-1)^{j} \frac{M_{i+j} a^{j}}{j !} \leqq M_{i}, \quad F(x)=\sum_{i=0}^{\infty} S_{i} \frac{x^{i}}{i !} .
$$

Now the function $F(x)$ so defined is an entire function. Let $x=b$. Then

$$
\begin{aligned}
F(b) & =\sum_{i=0}^{\infty} S_{i} \frac{b^{i}}{i !}=\sum_{i=0}^{\infty}(-1)^{i} \frac{M_{i}}{i !}\left(\sum_{j=0}^{i}(-1)^{i} C_{i-j, j} a^{i-j} b^{j}\right), \\
& =\sum_{i=0}^{\infty}(-1)^{i} \frac{M_{i}(a-b)^{i}}{i !} .
\end{aligned}
$$

And since the series

$$
\sum_{i=0}^{\infty} \frac{M_{i} a^{i}\left|(1-b / a)^{i}\right|}{i !}<M_{0} \sum_{i=0}^{\infty} \frac{\left|(1-b / a)^{i}\right|}{i !}
$$

converges, the series $\sum_{i=0}^{\infty}\left(S_{i} / i !\right) b^{i}$ converges. ${ }^{5}$ Further,

$$
F^{(i)}(a)=\sum_{j=0}^{\infty}(-1)^{i} M_{i+j} a^{j}\left(\sum_{k=0}^{j} \frac{(-1)^{k}}{k !(j-k) !}\right)=M_{i},
$$

and

$$
\left|F^{(i)}(x)\right| \leqq F^{(i)}(a)=M_{i}, \quad 0 \leqq x \leqq a .
$$

Thus we have given explicitly a function $F(x)$ satisfying the conditions of the theorem.

Armour Institute of Technology

5 See T. J. I'A. Bromwich, Theory of Infinite Series, London, 1931, p. 266. 